\title{
Nonalcoholic steatohepatitis: global impact and clinical consequences
}

\author{
Khaled Kabarra', Pegah Golabi² and Zobair M Younossi 1,2,3 \\ ${ }^{1}$ Center for Liver Diseases, Department of Medicine, Inova Fairfax Hospital, Falls Church, Virginia, USA \\ ${ }^{2}$ Betty and Guy Beatty Center for Integrated Research, Inova Health System, Falls Church, Virginia, USA \\ ${ }^{3}$ Inova Medicine, Inova Health System, Falls Church, Virginia, USA
}

Correspondence should be addressed to Z M Younossi: Zobair.Younossi@inova.org

\begin{abstract}
Nonalcoholic fatty liver disease (NAFLD) encompasses a spectrum of liver diseases ranging from simple fatty infiltration of liver parenchyma to the potentially progressive type of NAFLD called nonalcoholic steatohepatitis (NASH). Given the obesity epidemic, NAFLD and NASH have reached alarming levels globally. Recent data suggest that more than a quarter of the world population is affected by NAFLD; however, the disease prevalence is higher in certain patient population, that is, 55\% prevalence rate among patients with type 2 diabetes (T2DM). Besides T2DM, NAFLD is also closely related to other metabolic abnormalities, such as visceral obesity, hypertension, and hyperlipidemia. It has been suggested that stage of liver fibrosis is the most important factor associated with mortality among patients with NAFLD. Additionally, patients with T2DM have increased risk of adverse outcomes. In addition to these metabolic abnormalities, older age and some genetic factors could pose additional risks. Patients with NAFLD and NASH have significantly impaired health-related quality of life than the general population. There is also a growing economical impact of NAFLD and NASH on healthcare systems around the globe. Despite a number of promising regimens as treatment options, healthy lifestyle modification with diet and exercise remains at the core of management of NAFLD and NASH.
\end{abstract}

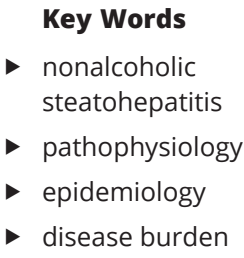

Endocrine Connections (2021) 10, R240-R247

\section{Introduction}

Nonalcoholic fatty liver disease (NAFLD) and its subtype of nonalcoholic steatohepatitis (NASH) were first described in the 1980s, coinciding with a rapid rise in epidemic of obesity $(1,2)$. Increasingly, NAFLD has been recognized as a liver complication of visceral obesity and other components of metabolic syndrome such as insulin resistance, diabetes, hypertension, and hyperlipidemia (3). In fact, NAFLD has recently been recognized as the most common cause of chronic liver disease (CLD), affecting more than a quarter of the world population (3).

In the past two decades, great strides have been made in the understanding of NAFLD. NAFLD is defined as the presence of hepatic steatosis affecting at least 5\% of liver hepatocytes in the absence of other causes of liver disease and excess alcohol consumption. The term NAFLD encompasses a spectrum ranging from histologic documentation of 'simple steatosis' (nonalcoholic fatty liver (NAFL)) to non-alcoholic steatohepatitis (NASH), which is histologic evidence of hepatic steatosis, lobular inflammation, hepatocyte ballooning with or without pericellular fibrosis, and/or Mallory-Denk bodies. NASH is considered the potentially progressive form of NAFLD that can progress to cirrhosis, hepatocellular carcinoma (HCC), and may require liver transplantation $(4,5)$ (Fig. 1).

The growing global burden of NAFLD has inspired research and expanded the understanding, not just of pathophysiology but also the burden it places on individuals, society, and the healthcare systems worldwide.
This work is licensed under a Creative Commons Attribution-NonCommercial 4.0 International License. ded from Bioscientifica.com at 04/26/2023 01:47:59PM 


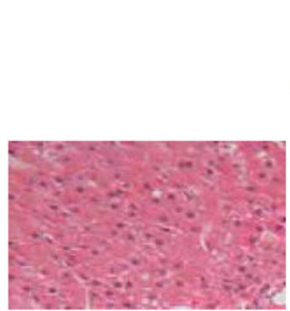

Normal

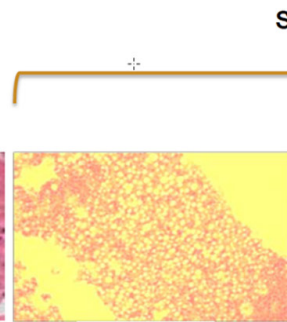

Steatosis
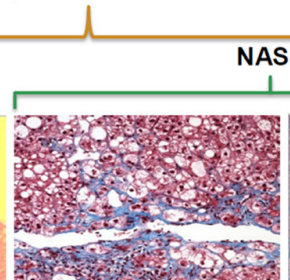

NASH
NASH

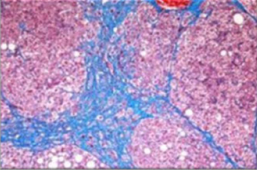

NASH with Advanced Fibrosis
Figure 1

NAFLD progression.
In this article, we focus on NAFLD/NASH and its clinical, economical, and patient-related burdens.

\section{Pathophysiology of NAFLD and NASH}

As noted previously, NASH is the potentially progressive form of NAFLD which requires early identification and linkage to care prior to development of significant liver disease, including advanced fibrosis and cirrhosis (Fig. 2). In fact, about $15-20 \%$ of patients with NASH can eventually develop cirrhosis (6). It is hypothesized that progression of NAFLD is linked to components of metabolic syndrome, especially visceral obesity and insulin resistance. Accumulation and dysregulation of lipid and glucose metabolism results in hepatic transcription factor dysfunction (7). This, as a result, creates a microenvironment that promotes inflammation and leads to hepatocyte ballooning and necrosis. This process can ultimately activate Stellate cells leading to accumulation of collagen and hepatic fibrosis in NASH. As fibrosis stage worsens, cirrhosis can be established which places patients at risk for liver failure and HCC (7).

Although NAFL is generally considered nonprogressive, a recent meta-analysis suggested that a few patients with NAFL and stage 0 fibrosis may progress over time (8). Factors associated with the progression of fibrosis in patients with NASH include hypertension, age, and low aspartate to alanine aminotransferase ratio. While patients with NASH will show 40\% fibrosis progression, some patients may be considered rapid progressors (8). Although the presence of increasing number of components of metabolic syndrome has been associated with advanced fibrosis and liver-related mortality, no other specific risk factor can currently identify 'rapid progressors' (9).

In the context of progressive liver disease, about $15-20 \%$ of NASH patients can progress to cirrhosis and $5-10 \%$ to end-stage liver disease (7). Other data have suggested that $41 \%$ of NASH individuals will develop progression fibrosis at a rate of $0.09 \%$ annually (3). One important complication of liver disease is HCC. In fact, HCC in NAFLD is associated with the presence of cirrhosis, older age, obesity, and the Patatin-like phospholipase domain-containing protein 3 (PNPLA3) gene (10). Data from Surveillance, Epidemiology, and End Result database suggest that NAFLD-related HCC is increasing by $9 \%$ yearly (11). It is important to note that HCC has also been described among NAFLD patients with metabolic syndrome without significant fibrosis or cirrhosis. These noncirrhotic NASH patients who develop HCC present a challenge and usually

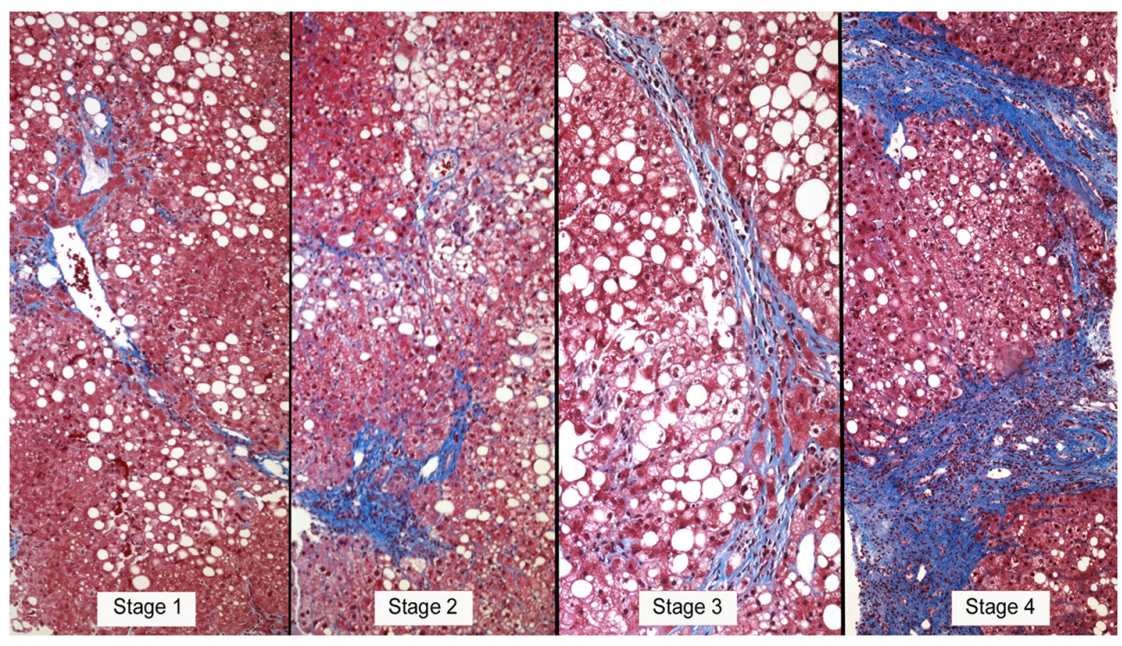

Figure 2

Masson's trichrome-stained sections showing stages of NASH progression. https://ec.bioscientifica.com https://doi.org/10.1530/EC-21-0048 (c) 2021 The authors Published by Bioscientifica Ltd

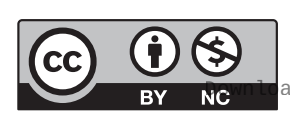

This work is licensed under a Creative Commons Attribution-NonCommercial 4.0 International License. ded from Bioscientifica.com at 04/26/2023 01:47:59PM 
present with more advanced disease possibly due to lack of proper and well-established surveillance. As a result, they tend to present with more advanced tumors with higher mortality and poorer prognosis as compared to HCC from viral hepatitis (12). It is important to note that the annual incidence of HCC among patients with NASH cirrhosis is around $0.5-2.6 \%$, while this rate is between 0.1 and 1.3 per 1000 patient-years among patients with non-cirrhotic NAFLD (13), which is below the accepted threshold for HCC screening.

\section{Modalities for hepatic fat detection}

Ultrasound (US), although it is used to detect hepatic steatosis, has low sensitivity and specificity especially in detecting lower grade of steatosis (14). Moreover, US only provides a qualitative rather than quantitative assessment of hepatic steatosis. Similarly, CT is suboptimal in detecting lower grade of hepatic steatosis (14). MRI on the other hand has the highest sensitivity and specificity in detecting even mild hepatic steatosis. Unfortunately, none of these imaging modalities can reliably distinguish between NASH and simple steatosis (15). Furthermore, these imaging modalities are unable to determine the stage of fibrosis in NASH. Although the presence of steatosis is required to establish the diagnosis of NAFLD, the grade of steatosis does not have prognostic value. On the other hand, establishing the diagnosis of NASH and determining stage of hepatic fibrosis have important prognostic value. In this context, despite a great deal of effort, none of the non-invasive tests (NITs) can establish the diagnosis of NASH which remains the basis of liver biopsy $(15,16,17)$.

Given the lack of NITs to establish diagnosis of NASH, the field has focused on developing validated NITs for hepatic fibrosis. In addition to novel radiological approach to assess liver stiffness and serum-based biomarkers of fibrosis, there have been multiple scoring systems developed to establish the presence of advanced hepatic fibrosis (15). These include aspartate aminotransferase to platelet ratio index, fibrosis-4 (FIB-4), and NAFLD fibrosis score (NFS). FIB-4 and NFS have shown to perform the best in excluding advanced fibrosis. These tests have good negative predictive value to exclude cirrhosis and may be valuable in clinical practice $(16,17)$. In addition to these algorithms, a number of serum biomarkers are being developed and validated. The biomarker that has been recently approved most is Enhanced Liver Fibrosis test (17). This test has excellent performance and is expected to be used as a part of algorithms to establish pathways to link high-risk NAFLD patients to care. Another test that is used in clinical research is PRO-C3, which detects collagen type 3 and may be useful in the future (16).

\section{Global prevalence of NAFLD}

It is estimated that global prevalence of NAFLD is about 25\% in the adult population with some geographic variation (3). An additional source of variability is diagnostic modalities used to establish the diagnosis of NAFLD. In fact, noninvasive serum tests, including liver enzyme elevation, may lead to lower prevalence rates for NAFLD. Nevertheless, the high and increasing prevalence of NAFLD seems to be proportionate with the prevalence of obesity, diabetes, and other metabolic diseases. Studies on incidence of NAFLD have been scant due to the lack of validated non-invasive modalities such as serum biomarkers that can be easily used in large-scale clinical and population-based studies.

The majority of the epidemiologic data in the Americas have been published from the United States $(18,19)$. Establishing the true prevalence of NASH is very difficult in the general population, given the need of histological confirmation. A study among military personnel and their dependents revealed an NAFLD prevalence of $46 \%$, with higher rates among Hispanics (58.3\%) and slightly lower rates among African Americans (35.1\%). Participants with positive US findings were offered a liver biopsy, and NASH prevalence was $29.9 \%$ among biopsied individuals and $12.2 \%$ in the total study cohort (20). However, given the referral and ascertainment biases, this study overestimates the prevalence of NASH. The impact of ethnicity on the prevalence of NAFLD has been considered. In this context, a study by Fleischman, in which NAFLD was diagnosed based on CT, reported an NAFLD prevalence of $29 \%$ in the whole cohort, 33\% among Hispanics of Mexican origin, $18 \%$ among Hispanics of Puerto Rican origin, and 16\% among Hispanics of Dominican origin (21).

In South America, epidemiological studies demonstrated higher rates of NAFLD prevalence. One study in which NAFLD was diagnosed with hepatic US, NAFLD prevalence was reported as $36 \%$. This rate was higher at $74 \%$ among individuals with metabolic syndrome and $73 \%$ among obese patients (22). Another study reported an NAFLD prevalence of $95 \%$ among obese individuals undergoing bariatric surgery, with an NASH prevalence of $66.7 \%$ with histologic evaluation (23).

The Middle East and Northern Africa (MENA) may have the highest prevalence of NAFLD globally with a rate of $32 \%$, and future projections demonstrate a worsening

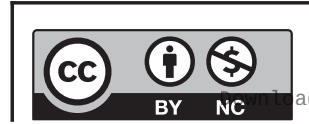

This work is licensed under a Creative Commons Attribution-NonCommercial 4.0 International License. ded from Bioscientifica.com at 04/26/2023 01:47:59PM 
trend (24). In this context, worsening diabetes and obesity rates have been shown to be the main driver of this trend in MENA countries. In fact, recent studies from the United Arab Emirates (UAE), Iran, or Turkey clearly demonstrate a disquieting trend in the rates of obesity, diabetes, and NAFLD $(25,26)$. For example, it is estimated that the number of patients with NAFLD will increase by $47 \%$ between 2017 and 2030 in the UAE, while the projection for NASH increase is 97\% in Saudi Arabia and $87 \%$ in UAE (). Due to this trend in the burden of NAFLD and NASH, it is anticipated that NASH will be the leading indication for liver transplantation in the Gulf countries in the next decades.

While the MENA region has the highest rates of NAFLD and NASH prevalence, the rest of the African continent is reported to have the lowest rate of around 13\% (3). Although some of this may be related to underreporting, lower prevalence of type 2 diabetes may also account for these lower rates. Nevertheless, similar to the rest of the world, type 2 diabetes and associated NAFLD prevalence is on the rise in many African countries.

Lastly, the growing burden of NAFLD in Asia is quite worrisome. In this context, many Asian countries are going through rapid transformation in their growing population and urbanization. This new rapid transformation and urbanization has led to changes in lifestyle, including diet and activity. Consequently, the rates of obesity, T2DM, and other components of metabolic syndrome are increasing. In this context, NAFLD burden in Asia is increasing at a rate that surpasses that in the Western countries and may become the leading cause of liver disease burden in the coming years (27). A study by Estes et al. projected a $30 \%$ increase in NAFLD prevalence by 2030 (28), which is likely due to worsening metabolic abnormalities. On the other hand, highly developed countries such as Japan and Singapore were able to demonstrate significantly better NAFLD rates than mainland China, as the rate of metabolic abnormalities was not as high in those high-income regions (29).

\section{Risk factors for NAFLD and NASH}

The close association between NAFLD and components of metabolic syndrome has been clearly demonstrated, and in fact, NAFLD is regarded as the hepatic manifestation of metabolic syndrome (30). Besides the prevalence and natural history of NAFLD and NASH, epidemiological studies have also been enlightening the risk factors for this common condition.
The most widely reported link to NAFLD and NASH is the components of metabolic syndrome (9). In this context, diabetes mellitus (DM) and NAFLD are particularly closely related. NAFLD prevalence is higher in patients with DM, and a recent meta-analysis reported that global prevalence of NAFLD among patients with type 2 DM was $55.5 \%$ (31). More strikingly, the global prevalence of NASH among patients with DM was 37.3\% in the same meta-analysis (31). It is known that the presence of DM in a patient with NAFLD increases the risk of NASH, advanced fibrosis, and mortality. Besides DM, hyperlipidemia, hypertension, and obesity are also common conditions in patients with NAFLD (3). As reported in the meta-analysis, NAFLD was present in $69 \%$ of patients with dyslipidemia, $51 \%$ of patients with obesity, and 39\% of patients with hypertension (3).

Age is also regarded as another risk factor for NAFLD and NASH. Both the prevalence of NAFLD and severity of hepatic fibrosis increase with age (32). Besides increased NAFLD risk, age is also a risk factor for complication of CLD, including liver mortality and HCC (33). On the other hand, there has been conflicting data about the association of gender with NAFLD. In this context, most recent studies have not suggested the predominance of a specific gender in development of progression of NAFLD and NASH.

Genetic predisposition to NAFLD development and progression among different racial and ethnic groups have also been studied previously. In this context, while some patients only develop NAFL, others may progress to NASH, advanced fibrosis, cirrhosis, or even death (34). Genome-wide association studies identified multiple DNA sequence variants, contributing to pathogenesis of hepatic steatosis (35). In this context, the most known associations are through PNPLA3 rs738409-G, glucokinase regulator (GCKR rs780094-T), and lysophospholipase-like 1 (LYPLAL1 rs12137855-C) (36).

\section{Clinical and economical burden of NAFLD}

\section{Clinical burden}

In 2019, CLD became the 11th leading cause of death, jumping from 12th in 2016 (37). As previously noted, NAFLD and NASH are the leading causes of CLD and have been on an upward trajectory to become the leading cause for liver transplantation (38).

In a meta-analysis that included four cohort studies, 1495 NAFLD patients and almost 17,500 patient-years of follow-up showed that with each increased stage of fibrosis,

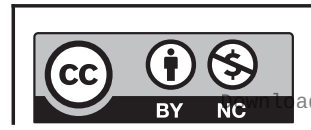

This work is licensed under a Creative Commons Attribution-NonCommercial 4.0 International License. ded from Bioscientifica.com at 04/26/2023 01:47:59PM 
the mortality (all-cause and liver-related) risk increased (39). In comparison to NAFLD without fibrosis (stage 0), NAFLD with fibrosis stages $1,2,3$, and 4 had a mortality risk ratio of $1.58,2.52,3.48$, and 6.40 , respectively (39). This illustrates the importance of identifying fibrosis as the single most important factor for predicting mortality.

NAFLD and NASH patients suffer from significant clinical, economical, and health-related quality of life (HRQoL) burden (40). It is widely known that patients with CLD suffer from significant impairment of HRQoL as compared to the general population (41). Fatigue, by far, is the most common symptom reported by patients with CLD, with a prevalence ranging between 50 and $85 \%$ (42). Besides being common, fatigue is also complex and challenging to treat. Patients suffering from fatigue are also suffering from daytime somnolence, inactivity, and physical function (43).

Other commonly reported impacts on quality of life include dietary limitations in more than half of the patients; 38\% reported impact on sleep, 31\% reported impact on social and family life, $29 \%$ reported healthcare frustration, and $26.5 \%$ were afraid of/worry about the future with particular concern about their disease getting worse (44). Patient-reported outcomes (PROs) assess the burden of disease by measuring patient's health status as reported by the patient without the interpretation of anyone else (45). One study reported that the key reported symptoms included upper abdominal pain, fatigue, and memory loss, while key HRQoL impacts included low quality of relationships, low mood, and self-consciousness (46). Given that NAFLD and NASH cause significant morbidity as well, recent research also focused on assessing the burden of disease by measuring disability-adjusted life years (DALY), which is the combination of years of life lost and years lived with disability. In this context, the Global Burden of Disease collaborators studied the global burden of cirrhosis over an almost 30-year period (47). This study reported that the number of prevalent cases for compensated cirrhosis due to NASH doubled over the study period, while the number for decompensated cirrhosis due to NASH tripled (47). Another study assessing the burden of NAFLD-/NASH-related burden using the same Global Burden of Disease database revealed that between 2007 and 2017, cirrhosis DALY due to NAFLD/NASH increased by $23.4 \%$, while liver cancer DALY due to NAFLD/NASH increased by $37.5 \%$ (48).

\section{Economical burden}

NAFLD/NASH also has an important economical burden that affects individuals, the healthcare system, and countries. The GAIN study analyzed the economical burden of NASH and NAFLD in five wealthy European countries, the Unites States, and China. The results showed that the mean total annual cost per patient with NASH in 2018 was $€ 2763$, €4917, and $€ 5509$ for direct medical, direct non-medical, and indirect costs, respectively. The United States exhibited the highest cost burden and France the lowest (49). The cost increased with the level of fibrosis and decompensation that required hospitalizations. Another study that extensively assessed the economical burden of NAFLD estimated that there were 64 million individuals with NAFLD in the United States and 52 million in four developed European countries (Germany, France, Italy, and UK), responsible for $\$ 103$ billion of direct costs in the United States, €27.7 billion in Germany, France, and Italy, and $£ 5.24$ billion in the UK (50). As stated above, individuals with diabetes are at an increased risk of NASH and adverse outcomes due to liver disease. Besides this fact, NAFLD/NASH patients with type 2 diabetes also suffer from a higher clinical and economical burden (51). The total economical burden for patients with NASH and DM is estimated to be $\$ 667.9$ billion in the United States, three quarters of which is attributed to DM care and the remaining $25 \%$ attributed to NASH care.

\section{Treatment options}

Currently, there are no specific pharmacologic agents that are approved by the food and drug administration to treat NAFLD/NASH. Existing therapies are directed toward NASH patients who are at risk for progressive liver disease. The gold standard treatment as recommended by most scientific societies is weight loss through calorie restriction and physical exercise (52). Body weight loss by $7 \%$ has been shown improvement on histology with steatohepatitis resolution in 90\% (53). Physical activity and calorie restriction remain the best method for weight loss in addition to the low cost of such intervention. As little as 60 min of exercise a week has shown benefits with $150 \mathrm{~min}$ a week showing optimal results (54). Moreover, evidence pointing at reducing calorie intake by $500=750 \mathrm{kcal} / \mathrm{day}$ has shown to reduce NASH fibrosis score (55). In addition, weight loss has shown to improve HRQoL (46). Current clinical practice guidelines suggest weight loss between 7 and 10\% of body weight through physical activity and calorie restriction (54).

If weight loss in 6-12 months is not achieved, some medical therapies can be considered (55). Of these, vitamin $\mathrm{E}$ has been shown to reduce hepatic steatosis, 
inflammation, ballooning, and improve activity (56). Given the strong association of diabetes with NAFLD, pioglitazone has also been used to treat NASH (56). The current evidence suggests that pioglitazone may be beneficial to some patients with histologically proven NASH (57). Other drugs such as pentoxifylline were initially promising, but current evidence does not support its use. For those who meet criteria, bariatric surgery or endoscopic procedures such as sleeve gastrectomy may be an option before development of cirrhosis (54). In patients with advanced cirrhosis or HCC, liver transplantation could be considered; however, morbid obesity may be an issue for these patients becoming candidates, especially in the presence of other comorbidities such as cardiovascular disease (54). In addition, NASH can recur despite liver transplant (54).

There are several other medications that are gaining interest in ongoing clinical trials (58). In fact, over the past decade, there has been a significant increase in registered clinical trials for NAFLD/NASH. Obeticholic acid, a selective farnesoid $\mathrm{X}$ receptor agonist that plays a role in lipid metabolism and inflammation, has been demonstrated by the REGENERATE trail to improve hepatic fibrosis (59). Liraglutide, a glucagon-like peptide 1 receptor agonist used for glycemic control in T2DM patients, has also shown in phase 2 clinical trials to reduce NASH with improvement in steatosis and hepatocyte ballooning (58). As stated above, these medications are in research process, and it is early to recommend these medications as a potential treatment option for NAFLD and NASH.

Finally, a combination of drugs that target different aspects of NASH pathogenesis may provide the best future therapeutic option for these patients. Given the chronic nature of NASH, it is important not only to enhance efficacy but also to ensure long-term safety of the approved regimens.

\section{Conclusions}

NAFLD/NASH continues to rise in alarming rate in conjunction with increase in the prevalence of various metabolic syndromes including diabetes. This phenomenon is also linked to the rapidly changing socio-economic landscape around the world. Hence, it is inevitable that NAFLD/NASH incidence will continue to rise worldwide. It has already become the leading cause of liver transplant. It is imperative to expect a growth in the burden of disease that parallels its trajectory. It is expected that with the continual increase in NAFLD/NASH rates, the economical burden will follow. The importance of being cognizant of the clinical and economical burden of NAFLD is paramount for the current and future management of this growing health problem.

\section{Declaration of interest}

$\mathrm{Z} M \mathrm{Y}$ has received research funds or served as consultant to Gilead Sciences, Intercept, NovoNordisk, BMS, Abbvie, Merck, Madrigal, Genfit, Siemens, Terns, and Viking. The other authors have nothing to report.

\section{Funding}

This work did not receive any specific grant from funding agencies in the public, commercial, or not-for-profit sectors.

\section{References}

1 Schaffner F \& Thaler H. Nonalcoholic fatty liver disease. Progress in Liver Diseases 19868 283-298. (available at: https://www.ncbi.nlm. nih.gov/pubmed/3086934)

2 Ludwig J, Viggiano TR, McGill DB \& Oh BJ. Nonalcoholic steatohepatitis: Mayo Clinic experiences with a hitherto unnamed disease. Mayo Clinic Proceedings 198055 434-438. (available at: https:// www.ncbi.nlm.nih.gov/pubmed/7382552)

3 Younossi ZM, Koenig AB, Abdelatif D, Fazel Y, Henry L \& Wymer M. Global epidemiology of nonalcoholic fatty liver disease-meta-analytic assessment of prevalence, incidence, and outcomes. Hepatology 2016 64 73-84. (https://doi.org/10.1002/hep.28431)

4 Estes C, Razavi H, Loomba R, Younossi Z \& Sanyal AJ. Modeling the epidemic of nonalcoholic fatty liver disease demonstrates an exponential increase in burden of disease. Hepatology 201867 123-133. (https://doi.org/10.1002/hep.29466)

5 Matteoni CA, Younossi ZM, Gramlich T, Boparai N, Liu YC \& McCullough AJ. Nonalcoholic fatty liver disease: a spectrum of clinical and pathological severity. Gastroenterology 1999116 1413-1419. (https://doi.org/10.1016/s0016-5085(99)70506-8)

6 Gramlich T, Kleiner DE, McCullough AJ, Matteoni CA, Boparai N $\&$ Younossi ZM. Pathologic features associated with fibrosis in nonalcoholic fatty liver disease. Human Pathology 200435 196-199. (https://doi.org/10.1016/j.humpath.2003.09.018)

7 Younossi Z, Anstee QM, Marietti M, Hardy T, Henry L, Eslam M, George J \& Bugianesi E. Global burden of NAFLD and NASH: trends, predictions, risk factors and prevention. Nature Reviews: Gastroenterology and Hepatology 201815 11-20. (https://doi. org/10.1038/nrgastro.2017.109)

8 Singh S, Allen AM, Wang Z, Prokop LJ, Murad MH \& Loomba R. Fibrosis progression in nonalcoholic fatty liver vs nonalcoholic steatohepatitis: a systematic review and meta-analysis of paired-biopsy studies. Clinical Gastroenterology and Hepatology 201513 643.e1-654. e9; quiz e39-e40. (https://doi.org/10.1016/j.cgh.2014.04.014)

9 Golabi P, Otgonsuren M, de Avila L, Sayiner M, Rafiq N \& Younossi ZM. Components of metabolic syndrome increase the risk of mortality in nonalcoholic fatty liver disease (NAFLD). Medicine 201897 e0214. (https://doi.org/10.1097/MD.0000000000010214)

10 Bugianesi E, Leone N, Vanni E, Marchesini G, Brunello F, Carucci P, Musso A, Paolis PD, Capussotti L, Salizzoni M, et al. Expanding the natural history of nonalcoholic steatohepatitis: from cryptogenic cirrhosis to hepatocellular carcinoma . Gastroenterology 2002123 134-140. (https://doi.org/10.1053/gast.2002.34168)

11 Younossi ZM, Otgonsuren M, Henry L, Venkatesan C, Mishra A, Erario $\mathrm{M} \&$ Hunt $\mathrm{S}$. Association of nonalcoholic fatty liver disease (NAFLD) with hepatocellular carcinoma (HCC) in the United States 
from 2004 to 2009. Hepatology 201562 1723-1730. (https://doi. org/10.1002/hep.28123)

12 Hester D, Golabi P, Paik J, Younossi I, Mishra A \& Younossi ZM. Among medicare patients with hepatocellular carcinoma, non-alcoholic fatty liver disease is the most common etiology and cause of mortality. Journal of Clinical Gastroenterology 202054 459-467. (https://doi. org/10.1097/MCG.0000000000001172)

13 Huang DQ, El-Serag HB \& Loomba R. Global epidemiology of NAFLDrelated HCC: trends, predictions, risk factors and prevention. Nature Reviews: Gastroenterology and Hepatology 202118 223-238. (https://doi. org/10.1038/s41575-020-00381-6)

14 Esterson YB \& Grimaldi GM. Radiologic imaging in nonalcoholic fatty liver disease and nonalcoholic steatohepatitis. Clinics in Liver Disease 201822 93-108. (https://doi.org/10.1016/j.cld.2017.08.005)

15 Golabi P, Sayiner M, Fazel Y, Koenig A, Henry L \& Younossi ZM. Current complications and challenges in nonalcoholic steatohepatitis screening and diagnosis. Expert Review of Gastroenterology and Hepatology 201610 63-71. (https://doi.org/10.1586/17474124.2016.109 9433)

16 Daniels SJ, Leeming DJ, Eslam M, Hashem AM, Nielsen MJ, Krag A, Karsdal MA, Grove JI, Guha IN, Kawaguchi T, et al. ADAPT: an algorithm incorporating PRO-C3 accurately identifies patients with NAFLD and advanced fibrosis. Hepatology 201969 1075-1086. (https:// doi.org/10.1002/hep.30163)

17 Younossi ZM, Corey KE, Alkhouri N, Noureddin M, Jacobson I, Lam B, Clement S, Basu R, Gordon SC, Ravendhra N, et al. Clinical assessment for high-risk patients with non-alcoholic fatty liver disease in primary care and diabetology practices. Alimentary Pharmacology and Therapeutics 202052 513-526. (https://doi. org/10.1111/apt.15830)

18 Sayiner M, Koenig A, Henry L \& Younossi ZM. Epidemiology of nonalcoholic fatty liver disease and nonalcoholic steatohepatitis in the United States and the rest of the world. Clinics in Liver Disease 2016 20 205-214. (https://doi.org/10.1016/j.cld.2015.10.001)

19 Younossi ZM, Stepanova M, Younossi Y, Golabi P, Mishra A, Rafiq N \& Henry L. Epidemiology of chronic liver diseases in the USA in the past three decades. Gut 202069 564-568. (https://doi.org/10.1136/ gutjnl-2019-318813)

20 Williams CD, Stengel J, Asike MI, Torres DM, Shaw J, Contreras M, Landt CL \& Harrison SA. Prevalence of nonalcoholic fatty liver disease and nonalcoholic steatohepatitis among a largely middleaged population utilizing ultrasound and liver biopsy: a prospective study. Gastroenterology 2011140 124-131. (https://doi.org/10.1053/j. gastro.2010.09.038)

21 Fleischman MW, Budoff M, Zeb I, Li D \& Foster T. NAFLD prevalence differs among hispanic subgroups: the multi-ethnic study of atherosclerosis. World Journal of Gastroenterology 201420 4987-4993. (https://doi.org/10.3748/wjg.v20.i17.4987)

22 Oni ET, Kalathiya R, Aneni EC, Martin SS, Blaha MJ, Feldman T, Agatston AS, Blumenthal RS, Conceiçao RD, Carvalho JAM, et al. Relation of physical activity to prevalence of nonalcoholic fatty liver disease independent of cardiometabolic risk. American Journal of Cardiology 2015115 34-39. (https://doi.org/10.1016/j. amjcard.2014.09.044)

23 Feijó SG, Lima JM, Oliveira MA, Patrocínio RM, Moura-Junior LG, Campos AB, Lima JW \& Braga LL. The spectrum of non-alcoholic fatty liver disease in morbidly obese patients: prevalence and associate risk factors. Acta Cirurgica Brasileira 201328 788-793. (https://doi. org/10.1590/s0102-86502013001100008)

24 Sanai FM, Abaalkhail F, Hasan F, Farooqi MH, Nahdi NA \& Younossi ZM. Management of nonalcoholic fatty liver disease in the Middle East. World Journal of Gastroenterology 202026 3528-3541. (https://doi.org/10.3748/wjg.v26.i25.3528)

25 Alswat K, Aljumah AA, Sanai FM, Abaalkhail F, Alghamdi M, Al Hamoudi WK, Al Khathlan A, Al Quraishi H, Al Rifai A, Al Zaabi M, et al. Nonalcoholic fatty liver disease burden - Saudi Arabia and United
Arab Emirates, 2017-2030. Saudi Journal of Gastroenterology 201824 211-219. (https://doi.org/10.4103/sjg.SJG_122_18)

26 Anushiravani A \& Ghajarieh Sepanlou S. Burden of liver diseases: a review from Iran. Middle East Journal of Digestive Diseases 201911 189-191. (https://doi.org/10.15171/mejdd.2019.147)

27 Wong MCS, Huang JLW, George J, Huang J, Leung C, Eslam M, Chan HLY \& Ng SC. The changing epidemiology of liver diseases in the Asia-Pacific region. Nature Reviews: Gastroenterology and Hepatology 201916 57-73. (https://doi.org/10.1038/s41575-018-0055-0)

28 Estes C, Anstee QM, Arias-Loste MT, Bantel H, Bellentani S, Caballeria J, Colombo M, Craxi A, Crespo J, Day CP, et al. Modeling NAFLD disease burden in China, France, Germany, Italy, Japan, Spain, United Kingdom, and United States for the period 2016-2030. Journal of Hepatology 201869 896-904. (https://doi.org/10.1016/j. jhep.2018.05.036)

29 Sarin SK, Kumar M, Eslam M, George J, Al Mahtab M, Akbar SMF, Jia J, Tian Q, Aggarwal R, Muljono DH, et al. Liver diseases in the Asia-Pacific region: a Lancet Gastroenterology and Hepatology Commission. Lancet: Gastroenterology and Hepatology 20205 167-228. (https://doi.org/10.1016/S2468-1253(19)30342-5)

30 Yki-Järvinen H. Non-alcoholic fatty liver disease as a cause and a consequence of metabolic syndrome. Lancet: Diabetes and Endocrinology 20142 901-910. (https://doi.org/10.1016/S22138587(14)70032-4)

31 Younossi ZM, Golabi P, de Avila L, Minhui Paik JM, Srishord M, Fukui N, Qiu Y, Burns L, Afendy A \& Nader F. The global epidemiology of NAFLD and NASH in patients with type 2 diabetes: a systematic review and meta-analysis. Journal of Hepatology 201971 793-801. (https://doi.org/10.1016/j.jhep.2019.06.021)

32 Vernon G, Baranova A \& Younossi ZM. Systematic review: the epidemiology and natural history of non-alcoholic fatty liver disease and non-alcoholic steatohepatitis in adults. Alimentary Pharmacology and Therapeutics 201134 274-285. (https://doi.org/10.1111/j.13652036.2011.04724.x)

33 Attar BM \& Van Thiel DH. Current concepts and management approaches in nonalcoholic fatty liver disease. ScientificWorldJournal 20132013 481893. (https://doi.org/10.1155/2013/481893)

34 Barbara M, Scott A \& Alkhouri N. New insights into genetic predisposition and novel therapeutic targets for nonalcoholic fatty liver disease. Hepatobiliary Surgery and Nutrition 20187 372-381. (https://doi.org/10.21037/hbsn.2018.08.05)

35 Sliz E, Sebert S, Würtz P, Kangas AJ, Soininen P, Lehtimäki T, Kähönen M, Viikari J, Männikkö M, Ala-Korpela M, et al. NAFLD risk alleles in PNPLA3, TM6SF2, GCKR and LYPLAL1 show divergent metabolic effects. Human Molecular Genetics 201827 2214-2223. (https://doi.org/10.1093/hmg/ddy124)

36 Anstee QM \& Day CP. The genetics of NAFLD. Nature Reviews: Gastroenterology and Hepatology 201310 645-655. (https://doi. org/10.1038/nrgastro.2013.182)

37 Asrani SK, Devarbhavi H, Eaton J \& Kamath PS. Burden of liver diseases in the world. Journal of Hepatology 201970 151-171. (https://doi. org/10.1016/j.jhep.2018.09.014)

38 Wong RJ, Aguilar M, Cheung R, Perumpail RB, Harrison SA, Younossi ZM \& Ahmed A. Nonalcoholic steatohepatitis is the second leading etiology of liver disease among adults awaiting liver transplantation in the United States. Gastroenterology 2015148 547-555. (https://doi.org/10.1053/j.gastro.2014.11.039)

39 Dulai PS, Singh S, Patel J, Soni M, Prokop LJ, Younossi Z, Sebastiani G, Ekstedt M, Hagstrom H, Nasr P, et al. Increased risk of mortality by fibrosis stage in nonalcoholic fatty liver disease: systematic review and meta-analysis. Hepatology 201765 1557-1565. (https://doi. org/10.1002/hep.29085)

40 Younossi ZM, Henry L, Bush H \& Mishra A. Clinical and economic burden of nonalcoholic fatty liver disease and nonalcoholic steatohepatitis. Clinics in Liver Disease 201822 1-10. (https://doi. org/10.1016/j.cld.2017.08.001)

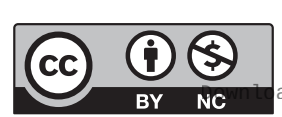

This work is licensed under a Creative Commons Attribution-NonCommercial 4.0 International License. ded from Bioscientifica com at 04/26/2023 01:47:59PM 
41 Younossi ZM, Boparai N, Price LL, Kiwi ML, McCormick M \& Guyatt G. Health-related quality of life in chronic liver disease: the impact of type and severity of disease. American Journal of Gastroenterology 2001 96 2199-2205. (https://doi.org/10.1111/j.1572-0241.2001.03956.x)

42 Gerber LH, Weinstein AA, Mehta R \& Younossi ZM. Importance of fatigue and its measurement in chronic liver disease. World Journal of Gastroenterology 201925 3669-3683. (https://doi.org/10.3748/wjg.v25. i28.3669)

43 Newton JL, Jones DEJ, Henderson E, Kane L, Wilton K, Burt AD $\&$ Day CP. Fatigue in non-alcoholic fatty liver disease (NAFLD) is significant and associates with inactivity and excessive daytime sleepiness but not with liver disease severity or insulin resistance. Gut 200857 807-813. (https://doi.org/10.1136/gut.2007.139303)

44 Younossi ZM, Golabi P \& Henry L. A comprehensive review of patientreported outcomes in patients with chronic liver diseases. Journal of Clinical Gastroenterology 201953 331-341. (https://doi.org/10.1097/ MCG.0000000000001179)

45 Doward LC, Balp MM, Twiss J, Slota C, Cryer D, Brass CA, Anstee QM $\&$ Sanyal AJ. Development of a patient-reported outcome measure for non-alcoholic steatohepatitis (NASH-CHECK): results of a qualitative study. Patient 202114 533-543. (https://doi.org/10.1007/s40271-02000485-w)

46 McSweeney L, Breckons M, Fattakhova G, Oluboyede Y, Vale L, Ternent L, Balp MM, Doward L, Brass CA, Beyer F, et al. Health-related quality of life and patient-reported outcome measures in NASH-related cirrhosis. JHEP Reports 20202 100099. (https://doi.org/10.1016/j. jhepr.2020.100099)

47 GBD 2017 Cirrhosis Collaborators, Safiri S, Bisignano C, Ikuta KS, Merat S, Saberifiroozi M, Poustchi H, Tsoi D, Colombara DV \& Abdoli A. The global, regional, and national burden of cirrhosis by cause in 195 countries and territories, 1990-2017: a systematic analysis for the Global Burden of Disease Study 2017. Lancet: Gastroenterology and Hepatology 20205 245-266. (https://doi.org/10.1016/S24681253(19)30349-8)

48 Paik JM, Golabi P, Younossi Y, Srishord M, Mishra A \& Younossi ZM. The growing burden of disability related to nonalcoholic fatty liver disease: data from the Global Burden of Disease 2007-2017. Hepatology Communications 20204 1769-1780. (https://doi.org/10.1002/ hep4.1599)

49 O’Hara J, Finnegan A, Dhillon H, Ruiz-Casas L, Pedra G, Franks B, Morgan G, Hebditch V, Jönsson B, Mabhala M, et al. Cost of nonalcoholic steatohepatitis in Europe and the USA: the GAIN study. JHEP Reports 20202 100142. (https://doi.org/10.1016/j.jhepr.2020.100142)
50 Younossi ZM, Blissett D, Blissett R, Henry L, Stepanova M, Younossi Y, Racila A, Hunt S \& Beckerman R. The economic and clinical burden of nonalcoholic fatty liver disease in the United States and Europe. Hepatology 201664 1577-1586. (https://doi.org/10.1002/hep.28785)

51 Younossi ZM, Tampi RP, Racila A, Qiu Y, Burns L, Younossi I \& Nader F. Economic and clinical burden of nonalcoholic steatohepatitis in patients with type 2 diabetes in the US. Diabetes Care 202043 283-289. (https://doi.org/10.2337/dc19-1113)

52 Chalasani N, Younossi Z, Lavine JE, Charlton M, Cusi K, Rinella M, Harrison SA, Brunt EM \& Sanyal AJ. The diagnosis and management of nonalcoholic fatty liver disease: practice guidance from the American Association for the Study of Liver Diseases. Hepatology 201867 328-357. (https://doi.org/10.1002/hep.29367)

53 Kleiner DE, Brunt EM, Van Natta M, Behling C, Contos MJ, Cummings OW, Ferrell LD, Liu YC, Torbenson MS, Unalp-Arida A, et al. Design and validation of a histological scoring system for nonalcoholic fatty liver disease. Hepatology 200541 1313-1321. (https://doi.org/10.1002/hep.20701)

54 Hung CK \& Bodenheimer HC Jr. Current treatment of nonalcoholic fatty liver disease/nonalcoholic steatohepatitis. Clinics in Liver Disease 201822 175-187. (https://doi.org/10.1016/j.cld.2017.08.012)

55 Marchesini G, Petta S \& Grave RD. Diet, weight loss, and liver health in nonalcoholic fatty liver disease: pathophysiology, evidence, and practice. Hepatology 201663 2032-2043. (https://doi.org/10.1002/hep.28392)

56 Sanyal AJ, Chalasani N, Kowdley KV, McCullough A, Diehl AM, Bass NM, Neuschwander-Tetri BA, Lavine JE, Tonascia J, Unalp A, et al. Pioglitazone, vitamin E, or placebo for nonalcoholic steatohepatitis. New England Journal of Medicine 2010362 1675-1685. (https://doi. org/10.1056/NEJMoa0907929)

57 Cusi K, Orsak B, Bril F, Lomonaco R, Hecht J, Ortiz-Lopez C, Tio F, Hardies J, Darland C, Musi N, et al. Long-term pioglitazone treatment for patients with nonalcoholic steatohepatitis and prediabetes or type 2 diabetes mellitus: a randomized trial. Annals of Internal Medicine 2016 165 305-315. (https://doi.org/10.7326/M15-1774)

58 Gawrieh S \& Chalasani N. Emerging treatments for nonalcoholic fatty liver disease and nonalcoholic steatohepatitis. Clinics in Liver Disease 201822 189-199. (https://doi.org/10.1016/j.cld.2017.08.013)

59 Ratziu V, Sanyal AJ, Loomba R, Rinella M, Harrison S, Anstee QM, Goodman Z, Bedossa P, MacConell L, Shringarpure R, et al. REGENERATE: Design of a pivotal, randomised, phase 3 study evaluating the safety and efficacy of obeticholic acid in patients with fibrosis due to nonalcoholic steatohepatitis. Contemporary Clinical Trials 201984 105803. (https://doi.org/10.1016/j.cct.2019.06.017)

Received in final form 27 August 2021

Accepted 6 September 2021

Accepted Manuscript published online 6 September 2021 https://ec.bioscientifica.com https://doi.org/10.1530/EC-21-0048 (c) 2021 The authors Published by Bioscientifica Ltd
This work is licensed under a Creative Commons Attribution-NonCommercial 4.0 International License. ded from Bioscientifica.com at 04/26/2023 01:47:59PM 